\title{
Photoacoustic imaging of breast cancer: a mini review of system design and image features
}

Nikhila Nyayapathi

Jun Xia 


\title{
Photoacoustic imaging of breast cancer: a mini review of system design and image features
}

\author{
Nikhila Nyayapathi ${ }^{\mathrm{a}, \mathrm{b}}$ and Jun $\mathrm{Xia}^{\mathrm{a}, *}$ \\ aUniversity at Buffalo, The State University of New York, Department of Biomedical Engineering, Buffalo, New York, United States \\ bUniversity at Buffalo, The State University of New York, Department of Electrical Engineering, Buffalo, New York, United States
}

\begin{abstract}
Breast cancer is one of the leading causes for cancer related deaths in women, and early detection is extremely important to improve survival rates. Currently, $x$-ray mammogram is the only modality for mass screening of asymptomatic women. However, it has decreased sensitivity in radiographically dense breasts, which is also associated with a higher risk for breast cancer. Photoacoustic (PA) imaging is an emerging modality that enables deep tissue imaging of optical contrast at ultrasonically defined spatial resolution, which is much higher than that can be achieved in purely optical imaging modalities. Because of high optical absorption from hemoglobin molecules, PA imaging can map out hemo distribution and dynamics in breast tissue and identify malignant lesions based on tumor associated angiogenesis and hypoxia. We review various PA breast imaging systems proposed over the past few years and summarize the PA features of breast cancer identified in these systems. (C) The Authors. Published by SPIE under a Creative Commons Attribution 4.0 Unported License. Distribution or reproduction of this work in whole or in part requires full attribution of the original publication, including its DOI. [DOI: 10.1117/1.JBO.24.12.121911]

Keywords: photoacoustics; breast imaging; biomedical optics; lasers.

Paper 190176SSRR received May 31, 2019; accepted for publication Oct. 14, 2019; published online Nov. 1, 2019.
\end{abstract}

\section{Introduction}

\subsection{Breast Cancer}

Breast cancer is the leading cause for cancer-related deaths in women all over the world. ${ }^{1}$ It occurs when cells in the breast start growing out of control due to accumulation of mutations in essential genes. ${ }^{2}$ Various genetic and environmental factors lead to such mutations and the growth of tumor mass. The mass can be asymptomatic or can sometimes be felt during a physical breast exam. The tumor is considered malignant when it starts to invade the surrounding tissue. Malignant breast cancer can present itself in many types, in which most are derived from the mammary ductal epithelium. Up to $75 \%$ of these are defined as invasive breast carcinoma, no special type (IDC-NST). Another type of epithelial carcinoma is called the invasive lobular carcinoma, which comprises about $5 \%$ to $15 \%$. Other rare types, such as tubular carcinoma, invasive cribriform carcinoma, and pure mucinous carcinoma ${ }^{3}$ constitute the rest. The WHO classification of tumors of the breast ${ }^{4}$ categorizes breast tumors and its subtypes. Sinn and Kreipe ${ }^{5}$ discussed and reviewed these in detail.

\subsection{Imaging of Breast Cancer}

Scientific studies have shown that breast cancer survival rates can be significantly improved with early detection. ${ }^{6-9}$ Therefore, it is key to detect breast cancer at an early stage, especially in asymptomatic women. X-ray mammogram is the standard imaging modality used for mass screening. However, it has decreased sensitivity in women with dense breast tissue. ${ }^{10}$ Since women with dense breasts are at an increased risk of breast cancer, ${ }^{11}$ there is a need for alternative screening methods. Magnetic resonance imaging (MRI) has good sensitivity and

*Address all correspondence to Jun Xia, E-mail: junxia@buffalo.edu specificity for dense breasts; ${ }^{12}$ however, this technique is very expensive and therefore not feasible as a mass screening procedure. Moreover, MRI requires the injection of Gadolinium as a contrast agent, which can lead to an allergic reaction, deposition in the central nervous system, or renal damage in certain cases. ${ }^{13-15}$ Although ultrasound (US) imaging is cost-effective and radiation-free, it has a high false positive rate and is often used in conjunction with mammogram. ${ }^{16-18}$ Hence, there is a need for a new imaging method that is not affected by breast density while overcoming the above-mentioned limitations.

\subsection{Principles of Photoacoustic Imaging}

Photoacoustics (PA) or optoacoustics, which uses optical excitation to generate ultrasonic waves, is a hybrid imaging modality with a high potential to solve the problems faced by existing imaging modalities, as mentioned in the previous subsection. Upon irradiation by pulsed laser light, biological tissues experience thermoelastic expansion, which leads to PA effect. ${ }^{19}$ The pressure waves that are generated can be detected using multiple US transducers. The acquired signal intensity and time of arrival can be used to reconstruct the strength and location of optical absorption in tissue. Since US scattering in tissue is 2 to 3 orders of magnitude less than optical scattering, ${ }^{20}$ the resolution achieved through this imaging technique is much higher than purely optical imaging modalities, such as diffuse optical tomography. ${ }^{21}$ With the use of near-infrared (NIR) light, PA imaging is also free of ionizing radiation.

Since different biological chromophores such as hemoglobin, water, lipids, and melanin have different absorption peaks, they can be differentiated using different wavelengths of light. In the short NIR region, hemoglobin is the major absorber in tissue. The development of microvascular structure due to tumor growth, called tumor angiogenesis, ${ }^{22}$ is a known biomarker for malignancy as rapidly growing tumors need nourishment to grow. ${ }^{23}$ Also as breast cancers start to grow over $2 \mathrm{~mm}$ in diameter, they are not adequately nourished due to the formation of 
disorganized vascular structures and leaky capillaries, ${ }^{24}$ which causes hypoxia. ${ }^{25}$ Average hemoglobin levels in tumor are reported to be higher in malignant tissue than in normal breast tissue. ${ }^{26}$ Therefore, most PA systems for breast cancer imaging focus on hemoglobin concentration and distribution as primary features to detect malignancy.

In this paper, we provide a review of various PA breast imaging systems and PA features for breast cancer identification. In Sec. 2, we discuss in detail the various components used to construct the system and to achieve the best possible imaging depth and spatial resolution in a fast and patient-friendly manner. We also summarize the advantages and drawbacks, and the imaging performance of each system. In Sec. 3, we discuss the PA image features of breast cancer identified by different systems. The final section gives an outlook of future directions in PA imaging of breast cancer.

\section{Imaging Systems}

As described in Xu and Wang, ${ }^{27} \mathrm{PA}$ imaging has three canonical detection geometries: spherical, cylindrical, and planar. The latter two geometries assume an infinitely tall or large cylindrical or planar detector arrays, respectively. The universal back projection algorithm was developed to offer exact reconstructions for these geometries. In practice, these three geometries are hard to achieve for breast imaging. For instance, it is impossible to cover the whole breast in a spherical detector and it is technically difficult to implement a large number of point-like detectors in cylindrical or planar geometries. In practical applications, the spherical geometry is implemented by scanning the object with a hemispherical detector, ${ }^{28}$ a spherical detector, ${ }^{29}$ or a curved array. ${ }^{30}$ The cylindrical detection is achieved by vertically scanning the object with a ring-shaped transducer. ${ }^{31,32}$ The planar detection is implemented using a small planar array ${ }^{33}$ or by scanning the object with a linear array. ${ }^{34}$ Apart from classification based on imaging geometry, the systems can also be categorized based on the scanning mechanism, i.e., handheld imaging systems and tomographic imaging systems. In handheld systems, the imaging probe can be used similar to a clinical breast US, whereas in tomographic imaging systems, the US probe is connected to a motorized stage for automated scanning. In the following section, we broadly classify the systems into two sections: tomography and handheld imaging systems. Within each section, we discuss the systems based on their imaging geometry.

\subsection{Tomographic Imaging Systems}

\subsubsection{Planar imaging systems}

The first planar view breast imaging system, the Twente Photoacoustic Mammoscope (PAM), was proposed by Piras et $\mathrm{al}^{33}$ In this system, the patient lies in a prone position with the breast pendant through an aperture in a patient bed. The breast is compressed with a glass plate for laser illumination on the cranial side and the US detector on the caudal side. Figure 1(a) illustrates a schematic of the system. A photograph of the breast against the detector is taken and the thickness after compression in the craniocaudal (CC) plane is measured. A flat array detector with 588 elements in an almost circular configuration is used. The size of each element in the array is $2 \times 2 \mathrm{~mm}^{2}$, with an element pitch of $3.175 \mathrm{~mm}$. The central frequency of the transducer is $1 \mathrm{MHz}$ and the field of view is $9 \times 8 \mathrm{~cm}^{2}$. A Q-switched Nd:YAG laser with 10 -ns pulse width, $10-\mathrm{Hz}$ pulse repetition rate, and 1064-nm output wavelength is used as the excitation source. The optical fluence on breast surface is $10 \mathrm{~mJ} / \mathrm{cm}^{2}$ and the light illumination area is $35 \mathrm{~cm}^{2}$. Using two eight-channel digitizers (60 MS/s, 12 bit), the authors acquired signals from a sequence of 10 elements at a time, and signals from each element was averaged by 100 times. The imaging time is about $10 \mathrm{~min}$ for the complete detector to cover an area of $9 \times 8 \mathrm{~cm}^{2}$ on the breast. ${ }^{35}$ The system has a spatial resolution of 3 to $4 \mathrm{~mm}$ in both lateral and axial directions. ${ }^{33,35-39}$ As shown in Fig. 1(a), the imaging depth for this system is measured from the cranial side of the breast in the sagittal plane. The maximum imaging depth that can be achieved by this system is $32 \mathrm{~mm} .{ }^{33}$ Figure 1 (b) shows an overlay of lesion identified by Twente PAM on CC x-ray images, obtained from a conventional $\mathrm{x}$-ray mammogram. The photo and compressed thickness of the breast are used for co-registration of PA and x-ray images in order to identify lesion location accurately. The image shows invasive ductal carcinoma (IDC) in a 69-year-old patient. The three-dimensional (3-D) representation of the lesion volume is shown in Fig. 1(c).

This system could successfully visualize most malignant tumors with high imaging contrast. The authors concluded that PA contrast at $1064 \mathrm{~nm}$ is mostly independent of breast density

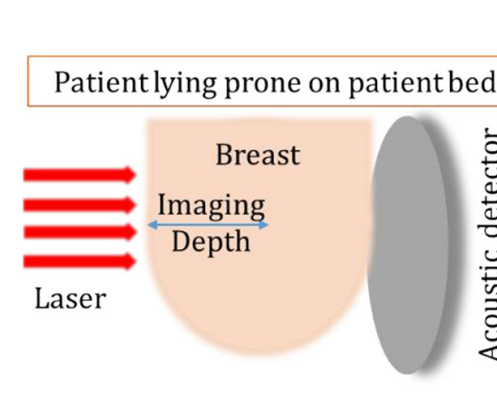

(a)

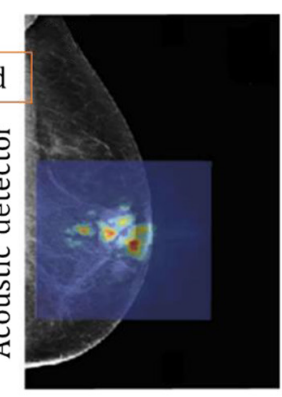

(b)

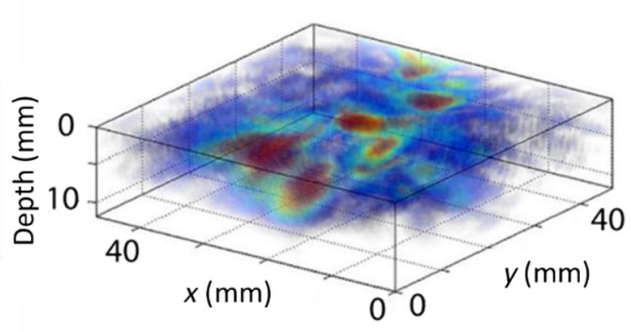

(c)

Fig. 1 (a) Twente PAM system. The patient lies in a prone position, with the breast pendant. The laser illuminates from the cranial side and the transducer detects on the caudal side of the breast. (b) Maximum intensity projection (MIP) PA image (color) overlaid on top of the left CC view x-ray mammogram (gray scale) of a 69-year-old patient with a 24-mm IDC (grade 2). (c) 3-D volume representation of the lesion region. This work by Heijblom et al. ${ }^{35}$ is licensed under a Creative Commons Attribution 4.0 International License. 
unlike in x-ray mammography. Because the breast is compressed in the CC plane as in an x-ray mammography, it is relatively easy to co-register Twente PAM and x-ray mammogram results. The use of a planar transducer array enables a uniform 3-D spatial resolution. However, similar to other PA systems, optical attenuation in tissue limits the imaging depth of Twente PAM. Since the laser is illuminated from only one side, the imaging depth of the Twente PAM is limited to $32 \mathrm{~mm}$ from the illuminated side of the breast [Fig. 1(a)]. Also, due to the use of single wavelength, the system cannot acquire functional information, such as oxygenated hemoglobin $\left(\mathrm{HbO}_{2}\right)$, deoxygenated hemoglobin $(\mathrm{Hb})$, or relative oxygen saturation levels $\left(\mathrm{sO}_{2}\right)$. Furthermore, activating only 10 elements at a time and the need of signal averaging lead to a relatively slow imaging time for the system.

The dual-scan mammoscope (DSM) proposed by Nyayapathi et al. is a double-sided-view planar imaging system. ${ }^{34}$ It uses two linear array transducers to scan the breast in the $\mathrm{CC}$ plane. The breast is gently compressed between two water tanks, with the patient standing upright. Two 128-element linear transducer arrays are mounted at right angles to the two outputs of a line output fiber bundle. These are held together by 3D-printed holders such that dichroic mirrors (97\% transmission at 45-deg illumination) could be placed at a 45-deg angle to both components. This design allows for coplanar light illumination and acoustic detection. A schematic of the system set-up is shown in Fig. 2(a). The central frequency of the transducers is $2.25 \mathrm{MHz}$, with element pitch of $0.7 \mathrm{~mm}$. A Q-switched $\mathrm{Nd}$ :YAG laser with $10-\mathrm{ns}$ pulse width, $10-\mathrm{Hz}$ pulse repetition rate, and 1064-nm output wavelength is used as the excitation source. The laser output is coupled to the circular input of a dual-line output fiber. The optical fluence on the skin surface is measured to be $21 \mathrm{~mJ} / \mathrm{cm}^{2}$. An imaging region of $8.6 \mathrm{~cm} \times$ $10 \mathrm{~cm} \times 7 \mathrm{~cm}$ is reported using this system. The latter two dimensions can be extended based on scanning length and breast thickness. The linear array is moved at a speed of $1 \mathrm{~mm} / \mathrm{s}$, thus scanning over a $6-\mathrm{cm}$ region takes $60 \mathrm{~s}$. For reconstruction, the universal back projection and 3-D focal-line reconstruction ${ }^{40}$ are used. The lateral and elevational resolutions are quantified to be 0.97 and $1.05 \mathrm{~mm}$, respectively. The data acquired from both transducers are combined and aligned to completely visualize the vascular structures in the compressed breast region. This is the first system to report PA imaging of human breast through a field of view of 7 -cm deep into the breast, ${ }^{34}$ as shown in Fig. 2(b). The image is projected in CC view, which is the orientation radiologists are most familiar with. The angiographic features agree with vascular structures from contrast-enhanced MRI. US data are also captured between PA pulses so that PA and US images are naturally co-registered for easy correlation. With double-sided illumination and detection, this planar system has a better depth visualization in comparison to single-sided planar imaging geometries. ${ }^{33,35-39,41-43}$ The imaging depth for this system is measured in the sagittal plane, as shown in Fig. 2(a). The maximum field of view achieved in the sagittal plane is $7 \mathrm{~cm}$ from both transducers combined.

Similar to the Twente PAM, the DSM system captures data in $\mathrm{CC}$ view, enabling easy correlate with $\mathrm{x}$-ray mammograms. Due to double-sided illumination, this system is successful in revealing vascular structures in the middle of the breast, with a combined imaging depth of $7 \mathrm{~cm}$ in the sagittal plane [Fig. 2(a)]. The use of a linear transducer array allows for easy implementation of US imaging, which could be useful in locating the suspicious lesion. Because the planar view is achieved by scanning the linear array, the field of view can be customized based on breast size. Limitations of the system include using a single wavelength $(1064 \mathrm{~nm})$, which prohibits functional characterization, and the nonuniform spatial resolution, due to the elevation resolution (along the scanning direction) being poorer than the axial or lateral resolutions of the imaging system using a linear array.

\subsubsection{Hemispherical imaging systems}

Kruger et al. ${ }^{44}$ proposed the first hemispherical detector array (HDA) for breast imaging. The first-generation HDA consists of 128 elements, 3-mm diameter each, with 5-MHz central frequency. The elements are laid out in a spiral pattern in a hemispherical surface of 100-mm radius of curvature. The rotation to multiple angular positions in a spiral distribution allows for an increase in k-space sampling density while maintaining uniform

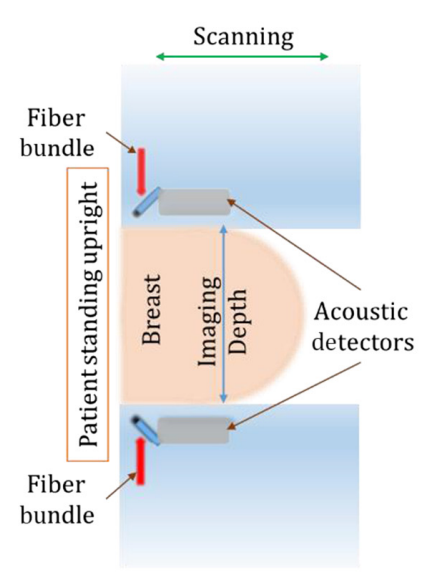

(a)

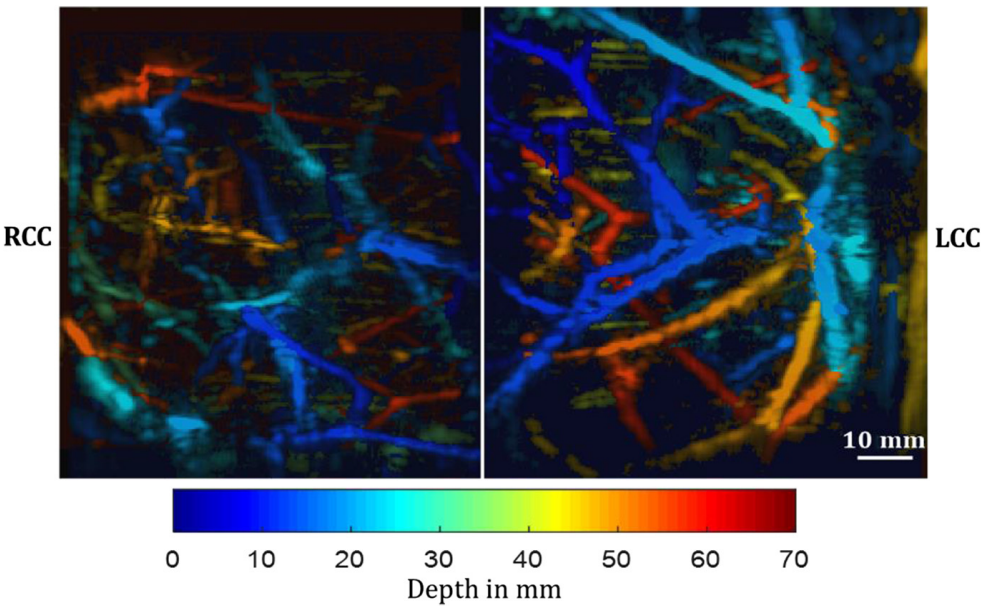

(b)

Fig. 2 (a) Schematic of DSM. (b) PA image of a volunteer with scattered fibroglandular breast density and thickness after compression of $7 \mathrm{~cm}$. Depth-encoded MIP formed by 3-D focal-line reconstruction algorithm. @ 2019 IEEE. Reprinted with permission. ${ }^{34}$ 


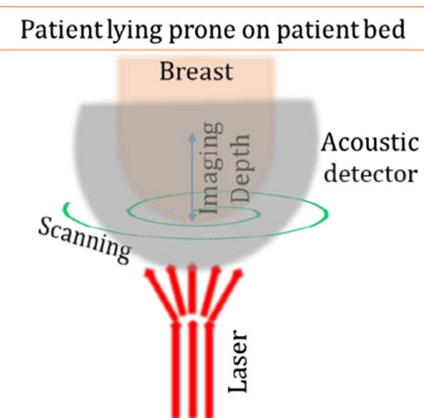

(a)

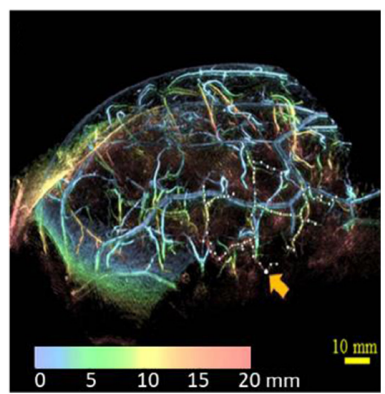

(b)
Fig. 3 (a) Schematic of the hemispherical (HDA) breast imaging system. The breast is placed inside the HDA cup. The whole array is scanned in a spiral pattern along the horizontal plane. (b) PA MIP image of a healthy breast. The orange arrow corresponds to the deepest point from skin surface at $27 \mathrm{~mm}$. This work by Toi et al. ${ }^{46}$ is licensed under a Creative Commons Attribution 4.0 International License.

angular sampling. In the subsequent generations, to increase the field of view, the rotational scanning is replaced by a rectilinear scan of the whole array in a spiral pattern. ${ }^{45-47}$ In addition, the number of the array elements is increased to 512, and the central frequency is reduced to $2 \mathrm{MHz}$. The spiral scan is such that PA data acquired is at equidistant locations. ${ }^{28,44,48}$ The advantage of hemispherical detection is that it allows for nearly spatially isotropic resolution in 3D. Figure 3(a) gives a schematic overview of the system geometry. The patient lies in prone position on the patient bed with the breast placed in a breast-holding cup of depth $38 \mathrm{~mm}$ placed above the HDA. A small amount of clean water is placed in the breast cup to allow for acoustic coupling between the breast and the breast cup. A Q-switched Alexandrite laser with selectable wavelengths of 755 and $795 \mathrm{~nm}$ is used to illuminate conically from the bottom of the cup in a $30-\mathrm{mm}$ radius through a $12-\mathrm{mm}$ diverging lens. The breast is compressed slightly toward the chest wall. Then the HDA scans the breast in a spiral pattern using $X Y$ translation stages. Scanning time for a single breast takes about $4 \mathrm{~min}$. The image is reconstructed using the universal back projection algorithm. The imaging depth for this system is measured from the surface of the breast touching the holding cup, radially inward. Toi et al. ${ }^{46}$ quantified the maximum depth by following the continuity of a blood vessel from the subcutaneous layer, deep into the breast. The deepest point from the skin surface is at $27 \mathrm{~mm}$, as marked by the orange arrow in Fig. 3(b). ${ }^{46}$

Since the array is hemispherical, this system eliminates the limited view problem that is prevalent in planar view systems and it provides a spatially isotropic resolution of $0.4 \mathrm{~mm}$. Multiwavelength imaging allows for the quantification of functional characteristics. However, similar to other single-sided illumination systems, this system has a small penetration depth of less than $30 \mathrm{~mm}$. The slow imaging time of 4 min per breast had caused motion artifacts that affected the calculation of $\mathrm{sO}_{2}$ despite applying motion correction processing. ${ }^{49,50}$ In addition, the system lacks intrinsic US imaging capability. So far, US imaging could only be achieved using a secondary transducer array, which needs additional image co-registration. ${ }^{47}$

Another hemispherical system implemented by Oraevsky et al. ${ }^{51}$ features an arc-shaped transducer that is rotated around the breast. This system, called the laser optoacoustic imaging system assembly (LOUISA-3D), consists of two subsystems- the PA subsystem and the US subsystem. The PA subsystem has a 90-deg arc-shaped array detector with 96 ultrawideband $(50 \mathrm{kHz}$ to $6 \mathrm{MHz}$ ) transducers. The patient lies on the examination bed with breast placed in a hemispherical plastic cup stabilizer. The complete breast is illuminated with a dual-pulsed Alexandrite laser (50-ns pulses) with toggling wavelengths of 757 and $797 \mathrm{~nm}$, separated by a time delay of 50 or $100 \mathrm{~ms}$. The short time delay is needed in order to accurately co-register the PA images and calculate functional images of total hemoglobin (HbT) and oxygen saturation in blood. The light is delivered using arc-shaped fiber segments in the radial direction toward the focal point of the arc. In order to maintain safe optical fluence of $20 \mathrm{~mJ} / \mathrm{cm}^{2}$, the system takes up to 10 steps to illuminate an entire breast (surface area $\sim 400 \mathrm{~cm}^{2}$ ), with beam area of $40 \mathrm{~cm}^{2}$. The imaging time per breast is about $10.6 \mathrm{~min}$ for a $10-\mathrm{Hz}$ interleaved scan. This accounts for two wavelengths, 10 steps per wavelength for full illumination of the breast, per transducer step for 320 angular positions. A spatial resolution of $\sim 0.3 \mathrm{~mm}$ can be achieved with this system. The US subsystem consists of another 90-deg arc-shaped transducer array with 192 transducers and $80-\mathrm{mm}$ radius. The central frequency is $7 \mathrm{MHz}$ with a wide bandwidth of $\pm 3.5 \mathrm{MHz}$. The 2-D slices of breast anatomy acquired by US subsystem are overlaid with corresponding PA slices from the 3-D images. The maximum imaging depth is $40 \mathrm{~mm}$, measured radially inward [Fig. 4(a).

In comparison to the hemispherical system in Fig. 3(a), the LOUISA-3D system achieves better imaging depth because the light illumination is provided at different angles through scanning the arc-shaped fiber. Other benefits of the system include high isotropic spatial resolution, full breast coverage, and dual-wavelength functional imaging. A challenge faced by this system is nonuniform illumination on the breast volume due to arc-shaped light delivery. Park et al. ${ }^{52}$ compensated for this by estimating the nonuniform illumination as a function of polar angle $\mathrm{e}^{20,53}$ and depth-dependent optical attenuation using Beer-Lambert law. ${ }^{54}$ An increase in penetration depth by $67 \%$ could be observed in comparison to noncompensated results. However, the acquisition takes over $10 \mathrm{~min}$, which may introduce motion artifacts. Although the system also acquires US data, it uses two different transducer arrays for US and PA imaging, which may further slow the imaging process.

\subsubsection{Cylindrical imaging systems}

Li et al. ${ }^{32}$ proposed a ring-shaped transducer array system named the functional photoacoustic tomography (fPAT). In fPAT, the patient lies in a prone position with the breast pendant through an aperture in the patient bed. The breast is mildly compressed into a cylindrical shape with diameter about $10 \mathrm{~cm}$ and thickness $<6 \mathrm{~cm}$. To achieve this, the ring array is adjusted to fit the breast. The transducer elements are moved radially inward and the polyethylene terephthalate glycol plastic plate is used to compress the breast toward the chest wall. The ring array consists of 64 detectors, arranged in 2 rows of 32 elements (element size $2.3 \times 30 \mathrm{~mm}$ ), based on a polyvinylidene fluoride film. The $-6-\mathrm{dB}$ bandwidth of each transducer is from $380 \mathrm{kHz}$ to $1.48 \mathrm{MHz}$ with a maximum frequency response up to $2 \mathrm{MHz}$. In order to cover the complete breast, the light is delivered from the bottom of the table through a concave lens and ground glass. A large illumination area is achieved by scanning the whole breast in 2-D using light delivery system. The transducers are connected to a 16 -channel preamplifier, and a $50-\mathrm{MHz}$ 


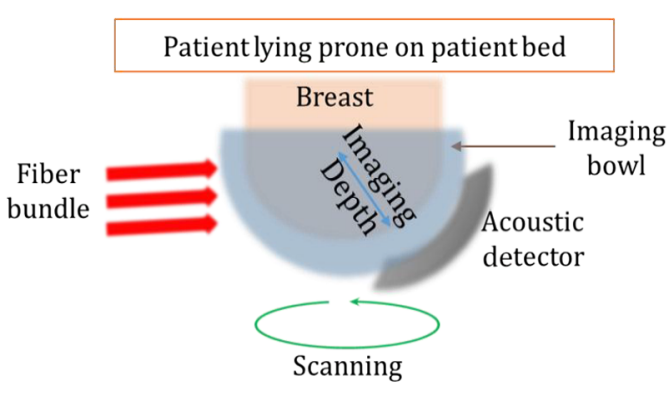

(a)

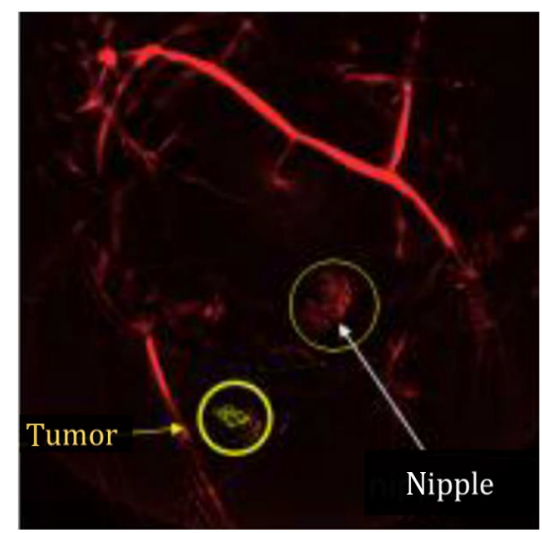

(b)

Fig. 4 (a) LOUISA 3-D schematic and (b) MIP in sagittal view of a 3-D PA image showing small tumorous growth and microvessel-filled nipple. The 3.5-mm tumor is not conclusively ascertained. Therefore, this is used to demonstrate the sensitivity of LOUISA-3D and not its specificity if tumor differentiation. Reproduced with permission. ${ }^{51}$

16-channel data acquisition board via mechanical switching. The diameter of the aperture is adjustable and can be fit to the size of the breast [Fig. 5(a)]. This system consists of a tunable pulsed Ti: sapphire laser, which is optically pumped with a Qswitched Nd:YAG laser. The pulse repetition rate is $10 \mathrm{~Hz}$ with a pulse width of 8 to $25 \mathrm{~ns}$. A $13 \mathrm{~mJ} / \mathrm{cm}^{2}$ energy density is generated by the light beam. The multispectral image reconstruction was performed using a total variation minimization-based finiteelement method to achieve deeper penetration depth. ${ }^{55}$ The resolution achieved by this system is $0.5 \mathrm{~mm} .{ }^{56}$ The functional characteristics used for fPAT diagnosis are calculated at the tumor sites as shown in Fig. 5(b). The increased hemoglobin concentration is observed for a lesion area of $1.9 \times 2.7 \mathrm{~cm}^{2}$ [Fig. 5(b)]. According to MRI, this region is located at $5 \mathrm{~cm}$ in the anterior-posterior dimension and $2.6 \mathrm{~cm}$ in $\mathrm{CC}$ dimension, and $5.6 \mathrm{~cm}$ posterior to the nipple. The lesion dimensions, measured by the full-width at half-maximum of $\mathrm{HbT}$ profiles, agree with the MRI report. The functional characteristics for the healthy (right) breast were shown in Fig. 5(c), where the distribution of $\mathrm{HbT}$ concentration and oxygen saturation are relatively uniform.

Compared to hemispherical imaging systems, the ring-array system can capture a cross-sectional image without moving the transducer array with a high spatial resolution of $0.5 \mathrm{~mm}$. The transducer array used in the fPAT system can be adjusted to fit different breast sizes. The use of multiple wavelengths further enables functional quantification of breast tissue. Also the complete breast is scanned in 2D with light delivery system for efficient illumination. However, this system does not capture US data, which may be useful in locating the tumor region. In addition, the transducer array has a $30-\mathrm{mm}$ slice thickness, which essentially limits the sectioning capability of the imaging system. Therefore, the exact location of the tumor in $3 \mathrm{D}$ is difficult to retrieve.

Lin et al. ${ }^{31}$ used a 512-element ring-shaped transducer array to achieve cylindrical imaging of the breast. In their single-breath-hold photoacoustic computed tomography (SBHPACT) system, the patient lies prone on a patient bed with breast suspended into a water tank through a hole in the table. The breast is slightly compressed toward the chest wall and scanned perpendicular to it as shown in Fig. 6(a). The detector is a 512element $2.25-\mathrm{MHz}$ ring transducer array, with $5-\mathrm{mm}$ element elevation height, $1.35-\mathrm{mm}$ element pitch, and $0.7-\mathrm{mm}$ interelement spacing. Four sets of 128-channel data acquisition systems are used to capture data from all 512 elements. A 1064-nm laser (10-Hz pulse repetition rate and 8- to $12-n s$ pulse width) light

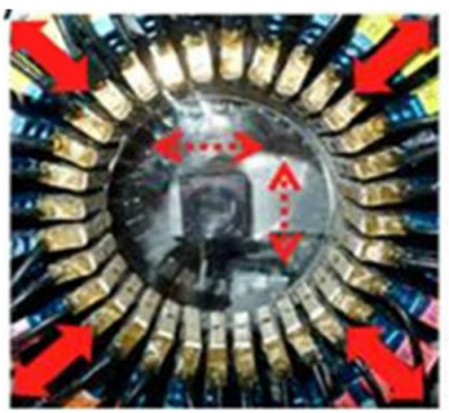

(a)

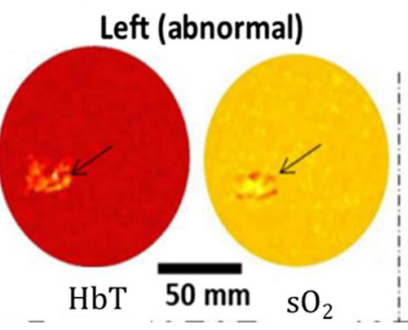

(b)

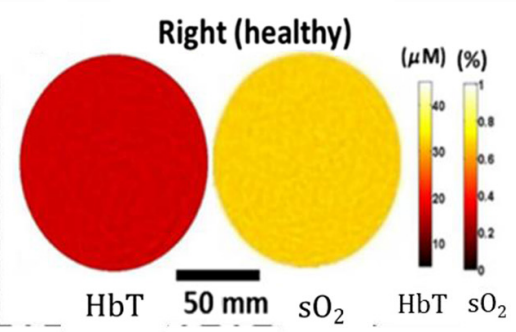

(c)

Fig. 5 (a) The top view of fPAT breast interface. Red solid arrows indicate that the diameter of the breast interface is radially adjustable; red dashed arrows indicate the path of the laser beam. (b) An invasive mammary carcinoma with high-grade ductal carcinoma in situ (DCIS) in the left breast. Coronal HbT concentration and $\mathrm{sO}_{2}$ (oxygen saturation) maps of a breast with invasive mammary carcinoma with high-grade DCIS. The arrows indicate the suspicious lesion area. (c) Coronal $\mathrm{HbT}$ and $\mathrm{sO}_{2}$ maps for the contralateral healthy breast. (c) $2015 \mathrm{Am}$. Assoc. Phys. Med. Reproduced with permission. ${ }^{32}$ 


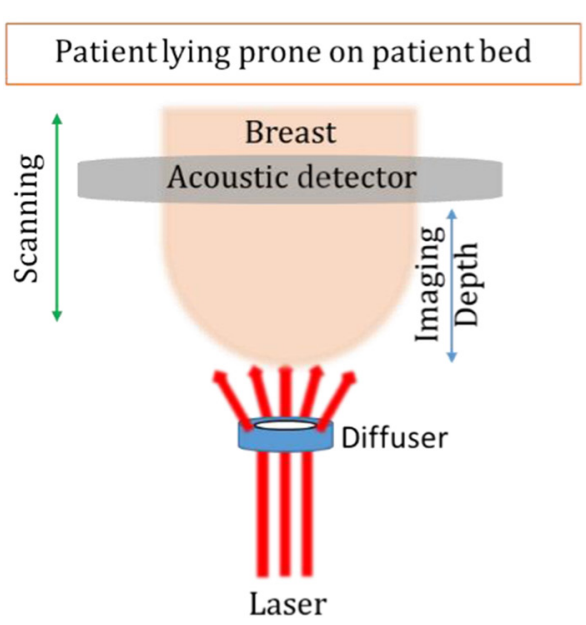

(a)
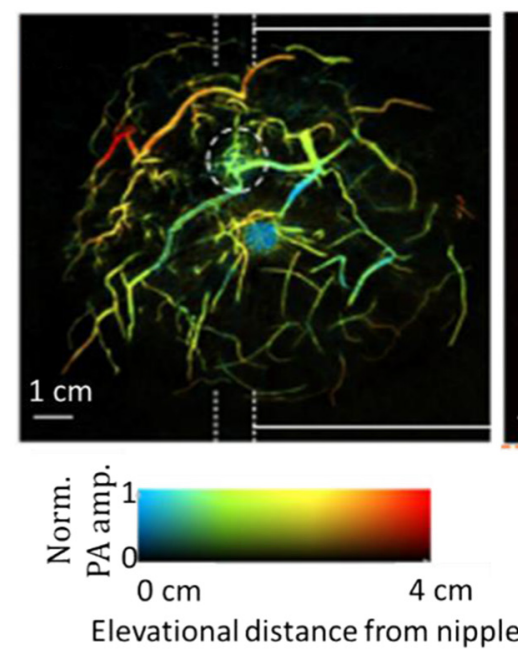

(b)

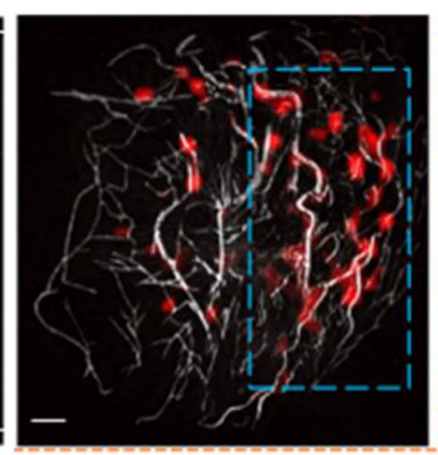

0

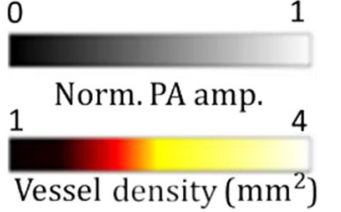

(c)

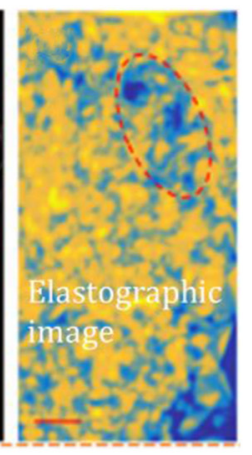

0.03

0.2

Relative area change

(d)

Fig. 6 (a) Schematic of the SBH-PACT system. The breast is scanned using a 512-element ring array transducer. The laser illuminates in a donut-shaped beam from the bottom. (b) Depth-encoded angiogram of a 44-year-old patient with fibroadenoma in the right breast acquired by SBH-PACT. The white dashed circle represents the tumor region. (c) PA MAP image of vessels deeper than the nipple of a 44-year-old female patient with an IDC in the left breast. Vessel density map (red) is overlaid on the MAP image but does not clearly demarcate the tumor region. (d) The PA elastographic image for the relative area change during breathing in blue dashed region in (c) is shown. Tumors are less compliant in comparison to normal breast tissue; therefore, they have smaller detectable relative area change when PA elastography is performed. The tumor could not be identified by SBH-PACT alone, however, elastographic study revealed the tumor marked by the red circle. This work by Lin et al. ${ }^{31}$ is licensed under a Creative Commons Attribution 4.0 International License.

is incident in a donut-shaped beam in order to achieve uniform illumination inside the breast. The entire scan lasts for an SBH ( $\sim 10$ to $15 \mathrm{~s}$ ), which eliminates breathing-induced motion artifacts. The image is reconstructed using the universal back projection algorithm. After 3-D image reconstruction, the elevational resolution is $5.6 \mathrm{~mm}$ and the in-plane resolution is $0.258 \mathrm{~mm}$. The maximum imaging depth achieved is $40 \mathrm{~mm}$, measured in the $\mathrm{CC}$ plane from the nipple toward the chest wall as shown in Fig. 6(a).

Figure 6(b) shows the depth-encoded angiogram acquired by SBH-PACT. The dashed circle represents the tumor region. Figure 6(c) shows the PA maximum amplitude projection (MAP) image (gray) and the corresponding vessel density map (red) of another breast. In this image, the tumor region is not easily identifiable. In order to improve the sensitivity of the system, the group also performed PA elastography. Since breast tumors are less compliant than normal breast tissue, when compression is applied, the tumor area would deform less than the normal breast tissue. In SBH-PACT, the compression is achieved through patient breathing, which compresses the breast against an agar pillow. After acquiring temporal image frames, the breast deformation is quantified by analyzing the change of area between blood vessels. With PA elastography, the system successfully identified a tumor [Fig. 6(d)] that can hardly be seen in the vessel density map [Fig. 6(c)]. The total time needed by SBH-PACT to simultaneously observe blood vessel density and breast tissue compliance is within $\sim 30 \mathrm{~s}$.

The SBH-PACT significantly reduces motion artifacts as the imaging itself occurs in a single breath-hold. With the addition of PA elastography, the system sensitivity is significantly improved with a negligible increase in imaging time. Also using a donut-shaped illumination allows for less energy deposition in the nipple and areola regions, which have higher concentrations of pigment. However, the illumination and detection schemes have limited the SBH-PACT's access to tumors close to the chest wall. Other limitations include the lack of US imaging capability and the use of a single wavelength.

\subsection{Handheld Systems}

\subsubsection{Linear-view imaging system}

Oraevsky et al. ${ }^{57}$ proposed a linear-array-based handheld PA system (Imagio) for functional imaging of breast. The system integrates diffused laser light through fiber bundles placed on either side and an US linear phased array transducer into a handheld probe [Fig. 7(a)]. Functional PA imaging of $\mathrm{HbO}_{2}$ and $\mathrm{Hb}$ is achieved using a dual-wavelength laser system made by Seno Medical Instruments. It comprises of a 15-ns pulsed Nd:YAG laser emitting $1064 \mathrm{~nm}$ wavelength and a 50-ns pulsed Alexandrite laser emitting $757 \mathrm{~nm}$ wavelength. In order to minimize the effect of tissue motion, the functional imaging is coregistered such that there is a 5-ms delay between pulses emitted at two wavelengths. The laser pulses are delivered in cycling pairs with a repetition rate of $5 \mathrm{~Hz}$. The transducer is a 128element linear array with element width of $0.25 \mathrm{~mm}$ and a wide bandwidth 0.1 to $12 \mathrm{MHz}$. The reconstruction method is a modified filtered back-projection algorithm ${ }^{58}$ that takes account of frequency response, angular response, and spatial impulse response of each transducer in the array. The axial resolution achieved is $0.47 \mathrm{~mm}$ for the Alexandrite laser (long-pulse width) and $0.42 \mathrm{~mm}$ for the Nd:YAG laser (short pulse width). Lateral resolution is measured to be the highest at the center of field and was $0.81 \mathrm{~mm}$ for the Alexandrite and $0.73 \mathrm{~mm}$ for the Nd:YAG laser. For objects located at $3 \mathrm{~cm}$ or farther from the probe and in 


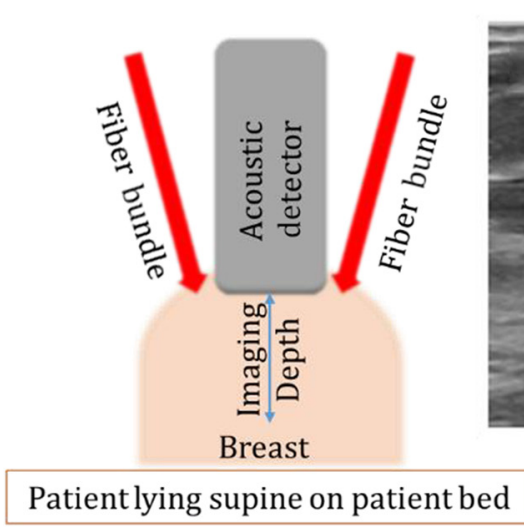

(a)

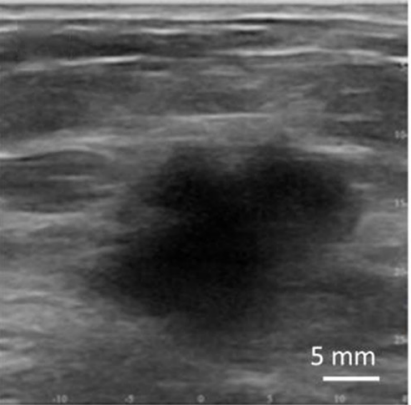

$\underline{\mathrm{mm}}$

)

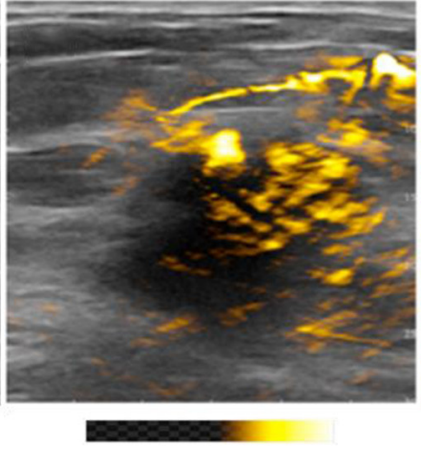

Functional PA map of $\mathrm{HbT}$

(c)

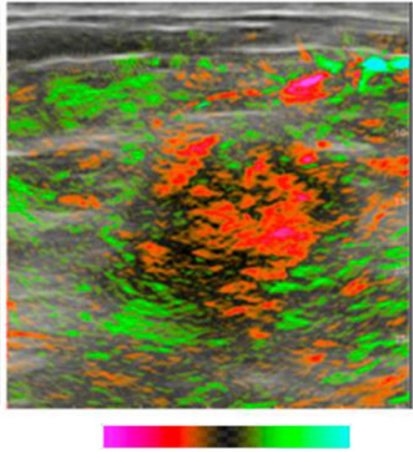

Functional PA map of $\mathrm{sO}_{2}$

(d)

Fig. 7 (a) A schematic of the handheld Imagio system. (b) Gray-scale US image of a 2.6-cm irregular intensely hypoechoic solid mass with indistinct margins. The mass is suspicious for malignancy. (c) Overlaid image of US and PA HbT map shows increased internal HbT concentration in more than half of the tumor region and a major vessel above the tumor. (d) Overlaid image of US and $\mathrm{PA} \mathrm{sO}_{2}$ map shows diffused internal blood deoxygenation, with numerous radiating parasitized feeding arteries (green) in tissue that surround the mass. The entire mass is identified as predominantly hypoxic. The mass was biopsied later and the histology revealed grade III IDC. This work by Oraevsky et al. ${ }^{57}$ is licensed under a Creative Commons Attribution 4.0 International License.

close proximity to the probe, the lateral resolution decreased to $\sim 1 \mathrm{~mm}$ for Alexandrite laser and $\sim 0.92 \mathrm{~mm}$ for $\mathrm{Nd}$ :YAG laser. ${ }^{57}$ At a video frame rate of $10 \mathrm{PA} / \mathrm{US}$ images per second, co-registered functional PA and B-mode US images are displayed simultaneously in real time. An example of a B-mode US image from the system is shown in Fig. 7(b), where a hypoechoic solid mass is shown at the center. Figure 7(c) quantifies the relative $\mathrm{HbT}$ concentration within and around the tumor region. Dense areas of tumor angiogenesis-related microvasculature and major vasculature are represented in yellow. The $\mathrm{sO}_{2}$ map of the same tumor is shown in Fig. 7(d), where the green color represents the typical $\mathrm{sO}_{2}$ level in normally oxygenated tissues, and the red color represents below normal $\mathrm{sO}_{2}$ level, which is a typical indication of malignancy [Fig. 7(d)]. The authors conclude that, using functional parameters such as $\mathrm{HbT}$ concentration and $\mathrm{sO}_{2}$, the specificity of diagnostic US can be enhanced.

The Imagio system has a high translational potential, because the system operation is similar to that of a breast US. The use of two wavelengths enables functional characterization of breast tissue, which improves the system's specificity. However, compared to tomographic imaging systems, the handheld system is operator-dependent and it cannot provide 3-D information of the tumor.

\subsubsection{Spherical array-based imaging system}

Dean-Ben et al. ${ }^{29}$ proposed a spherical-array volumetric handheld scanner for real-time PA imaging in dense breast. A custommade 256-element 2-D array of detectors is arranged on a spherical surface with radius $40 \mathrm{~mm}$ and covering a solid angle of $90 \mathrm{deg}$. The central frequency of the transducer is $4 \mathrm{MHz}$ with a size of $3 \mathrm{~mm} \times 3 \mathrm{~mm}$. A wavelength-tunable optical parametric oscillator laser ranging from 690 to $900 \mathrm{~nm}$ emits $<10 \mathrm{~ns}$ pulses with a pulse repetition rate of $10 \mathrm{~Hz}$. Three wavelengths of 730 , 760 , and $850 \mathrm{~nm}$ are used. The light is delivered to the center of the detection array through a fused silica fiber bundle for coaxial illumination as shown in Fig. 8(a). A transparent polyethylene membrane is used to enclose the active detection surface. To ensure acoustic coupling, the volume in between is filled with water. For data acquisition, 256 parallel analog to digital converters are arranged in 16 acquisition cards with 16 channels each, and a sampling rate of $40 \mathrm{MS} / \mathrm{s}$ is employed. The spatial resolution is quantified to be in the range of $200 \mu \mathrm{m} .{ }^{59}$ The reconstruction is performed using a 3-D model-based procedure for spectral unmixing. ${ }^{60}$ Distribution maps for oxygenated and deoxygenated hemoglobin and melanin are obtained by least-square fitting the reconstructed optical absorption in each image voxel to the known molar extinction coefficient of the corresponding tissue chromophores [Fig. 8(b)]. The angiographic features of the breast are visualized up to a depth of $22 \mathrm{~mm}^{29}$

The spherical transducer array allows acquisition of 3-D volumetric images in real time with a single laser shot and it minimizes the limited-view problem. The fast wavelengthswitching laser enables multispectral imaging at a high speed. However, the system is also operator dependent and the imaging depth is only about $22 \mathrm{~mm}$, which may pose a problem in locating deep-seated lesions.

\subsubsection{Curved array-based imaging system}

Diot et al. ${ }^{30}$ proposed a curved-array handheld multispectral optoacoustic tomography (MSOT) system with 28 wavelengths. The 256-element transducer array has a diameter $120 \mathrm{~mm}$ and central frequency of $5 \mathrm{MHz}$. The transducer elements are arranged in an arc spanning $174 \mathrm{deg} .{ }^{61}$ The transducer is enclosed in a transparent low-density polyethylene membrane, with the cavity between the arc and membrane filled with heavy water $\left(D_{2} O\right)$ for acoustic coupling as shown in Fig. $9(a)$. $D_{2} O$ is used because it absorbs less light than water in the used wavelength range. ${ }^{62}$ A tunable pulsed laser with a working range of 680 to $980 \mathrm{~nm}$, pulse length of $8 \mathrm{~ns}$, and repetition rate of $50 \mathrm{~Hz}$ is coupled with a custom made line output $\left(40 \times 1 \mathrm{~mm}^{2}\right)$ fiber. For data acquisition, a custom-built analog-to-digital converter is used at a sampling rate of $40 \mathrm{MS} / \mathrm{s}$ and 12-bit digital resolution. A delay and sum reconstruction algorithm is used for real-time viewing. The patient is scanned in a supine position. The tumor is first located using conventional US, and then the area is 


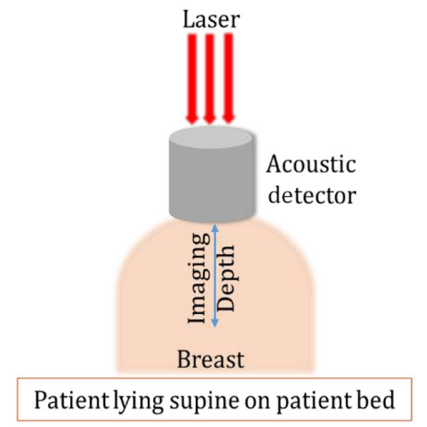

(a)

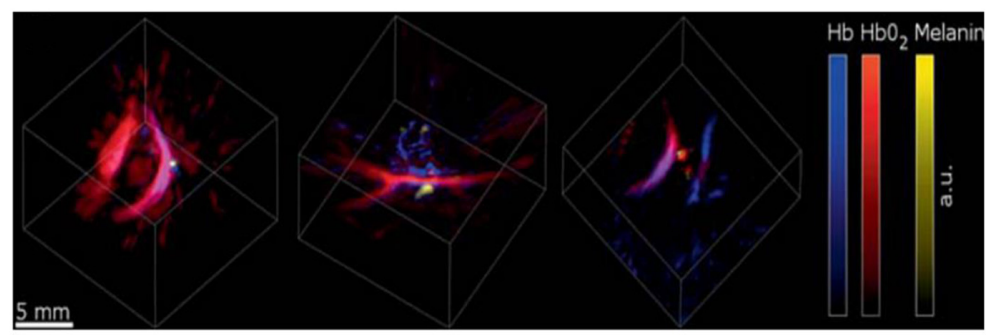

(b)

Fig. 8 (a) Schematic of the volumetric 3-D handheld scanner. (b) Results from functional PA breast angiography using multiwavelength data acquisition as it simultaneously resolves major tissue chromophores in the breast of a 40-year-old volunteer in 3D, in real time. The breast surface is located in the upper part of the cubes. $\odot 2015$ by Wiley-VCH. Reproduced with permission. ${ }^{29}$

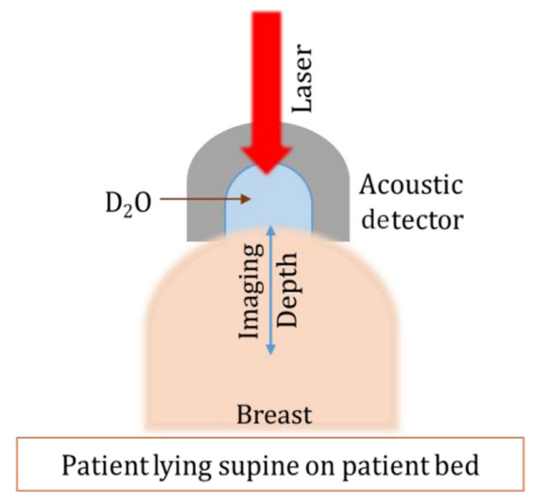

(a)

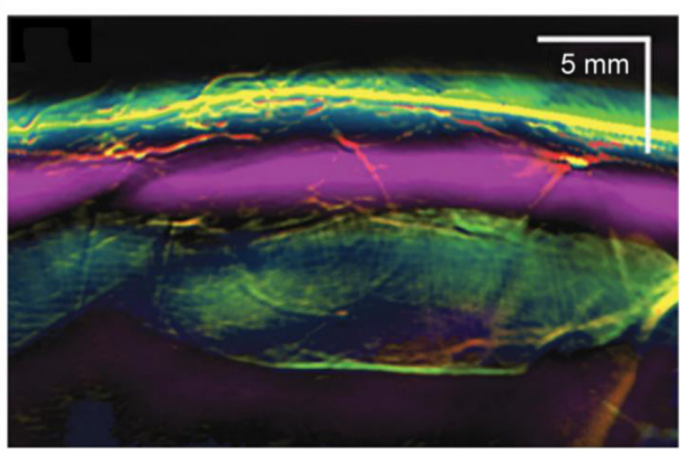

(b)

Fig. 9 (a) A schematic of the handheld MSOT probe. (b) Results show a composite image of all four absorbers $\left(\mathrm{Hb}, \mathrm{HbO}_{2}\right.$, lipid, and water), revealing the layered structure of the breast. (Yellow, skin; pink, lipid; light blue, mammary tissue; green, connective tissue known as Cooper ligament). ( 2017 AACR. Reprinted with permission. ${ }^{30}$

scanned by handheld MSOT. During the scan, slight pressure is applied to reduce tumor depth. For each cross-sectional slice, 28 frames are collected at wavelengths ranging from 700 to $970 \mathrm{~nm}$ in $10 \mathrm{~nm}$ steps. Each multiwavelength slice is acquired in $\sim 0.56 \mathrm{~s}$, with a total examination time of 2 to $4 \mathrm{~min}$. MSOT can achieve a high spatial resolution of $\sim 250 \mu \mathrm{m},{ }^{63}$ but it is limited to a penetration depth of 2 to $3 \mathrm{~cm}$ due to light attenuation. ${ }^{62}$ After spectral unmixing is applied to all 28 wavelengths, four images are produced representing the absorption of $\mathrm{HbO}_{2}, \mathrm{Hb}$, lipid, and water. Figure 9(b) shows the composite image of all four absorbers in breast tissue. The high spectral resolution of MSOT due to multiple wavelengths allowed calculating the total blood volume (TBV) gradient, which measures the spatial heterogeneity from periphery to the center of the tumor. Using TBV gradient as the metric, authors observed fivefold variability in between tumors. The authors suggested that TBV gradient could be used to differentiate various tumor subtypes.

The handheld MSOT provides information about physiological features of cancer. With the use of 28 wavelengths and generation of four functional maps, this system has a unique capability to analyze blood oxygenation levels, blood volume, lipid, and water behavior in the tumor region with improved quantitative accuracy. The authors state that TBV gradient characteristics could be used to differentiate tumor subtypes.
However, being a handheld device, the MSOT is operatordependent and provides only 2-D tumor information. In addition, prior to imaging with handheld MSOT, the tumor region has to be located using a conventional US system.

\subsection{Summary}

As mentioned above, a wide variety of system geometries have been proposed for PA imaging of human breast. The characteristics of the systems are summarized in Table 1.

\section{PA Features for Breast Imaging}

To provide useful insights for the future of PA imaging of breast cancer, in Table 2, we summarize the PA characteristics identified through various imaging studies.

One of the main functional characteristics for tumor diagnosis is oxygen saturation [Fig. 10(a)]. Aggressively growing tumors are not adequately nourished, leading to hypoxic conditions (lower amount of oxygen saturation) in the tumor mass. Since oxygenated hemoglobin and deoxygenated hemoglobin have different peaks in the NIR region, two wavelengths can be used to measure the oxygen saturation levels. Li et al., ${ }^{32}$ 
Table 1 System characteristics.

\begin{tabular}{|c|c|c|c|c|c|c|}
\hline System & $\begin{array}{l}\text { Patient } \\
\text { position }\end{array}$ & $\begin{array}{c}\text { Imaging } \\
\text { depth (mm) }\end{array}$ & Spatial resolution & $\begin{array}{c}\text { US } \\
\text { imaging }\end{array}$ & $\begin{array}{l}\text { Imaging } \\
\text { time/speed }\end{array}$ & $\begin{array}{l}\text { Multispectral } \\
\text { imaging }\end{array}$ \\
\hline Single-sided planar-view ${ }^{33}$ system & Prone & 35 & $3 \mathrm{~mm}$ & No & $10 \mathrm{~min}$ & No \\
\hline Double-sided planar-view system ${ }^{34}$ & Upright & $70^{\mathrm{a}}$ & $\begin{array}{l}0.9 \mathrm{~mm} \text { in lateral } \\
1 \mathrm{~mm} \text { in elevation }\end{array}$ & Yes & $1 \mathrm{~min}$ & No \\
\hline Hemispherical array-based system ${ }^{46}$ & Prone & 27 & $0.4 \mathrm{~mm}$ & No & $4 \min$ & Yes \\
\hline Arc-array-based hemispherical view system ${ }^{51}$ & Prone & 40 & $0.3 \mathrm{~mm}$ & Yes & $10 \mathrm{~min}$ & Yes \\
\hline Ring array-based system ${ }^{32}$ & Prone & - & $0.5 \mathrm{~mm}$ in-plane & No & - & Yes \\
\hline Ring-shaped array system ${ }^{31}$ & Prone & $40^{b}$ & 0.258 in-plane & No & $15 \mathrm{~s}$ & No \\
\hline Handheld linear array ${ }^{57}$ & Supine & 30 & & Yes & $10 \mathrm{~Hz}$ & Yes \\
\hline Handheld spherical array ${ }^{29}$ & Supine & 22 & $200 \mu \mathrm{m}$ around the center & No & $10 \mathrm{~Hz}$ & Yes \\
\hline Handheld arc-shaped array ${ }^{30}$ & Supine & 20 to 30 & $250 \mu \mathrm{m}$ & No & $2 \mathrm{~Hz}$ & $Y_{e s}{ }^{c}$ \\
\hline
\end{tabular}

${ }^{a}$ Combined depth from two transducers.

${ }^{\mathrm{b}}$ Depth measured from the nipple region.

${ }^{\mathrm{c}} 28$ wavelengths.

Oraevsky et al., ${ }^{57}$ Dean-Ben et al., ${ }^{29}$ and Diot et al. ${ }^{30}$ successfully demonstrate this possibility in their imaging systems.

Another important functional characteristic is HbT levels [Fig. 10(b)]. The hypoxia in tumors induces expression of vascular endothelial growth factor, which leads to growth of vessels

Table 2 PA features identified and their significance for breast cancer.

Features identified

Why these features are important

Oxygen saturation

Tumor regions are prone to hypoxia as rapidly growing tumor cells are undernourished due to the formation of leaky capillaries. Visualization of intratumoral hemoglobin oxygenation status is useful in order to monitor anticancer treatments noninvasively

HbT concentration

Fast growing tumors are associated with higher levels of blood in comparison to normal tissue. Therefore, $\mathrm{HbT}$ concentration levels are indicative of tumor microvasculature

VBPs

Comparison of VBPs in the tumor region and contralateral healthy tissue can indicate potential cancer identifying feature

Centripetal vasculature/ Tumor-related blood vessels seem to peripheral vascularity converge toward the center of the tumor, becoming drastically narrower at the tumor edge and nearly vanishing at the center. This is indicative of tumor angiogenesis and a hallmark of cancer development

Vessel density Higher number of vessels in the tumor region due to tumor angiogenesis

Relative area change Differentiation of healthy breast tissue (PA elastography) from tumor areas based on rigidity with leaky capillaries. ${ }^{64}$ This leads to higher levels of hemoglobin in the tumor region than that of normal tissue. $\mathrm{Li}$ et al., ${ }^{32}$ Oraevsky et al., ${ }^{57}$ Dean-Ben et al., ${ }^{29}$ and Diot et al. ${ }^{30}$ demonstrated the use of $\mathrm{HbT}$ level to identify breast tumor.

Based on the concept that tumor angiogenesis is a biomarker for malignancy, 22 areas of high vascular densities could be associated with rapidly growing tumors [Fig. 10(e)]. Using singlewavelength studies, Heijblom et al. ${ }^{35}$ (lower resolution) and Lin et al. ${ }^{31}$ (higher resolution) identified regions with a higher vessel density, which were confirmed to be tumors.

Yamaga et al. ${ }^{45}$ proposed vascular branching points (VBPs) as a possible biomarker for breast cancer [Fig. 10(c)]. An increase could be observed in the number of VBPs in the subcutaneous region of the tumor breast when compared with contralateral healthy breast. This feature is derived from the characteristic that malignant tumor is associated with sustained angiogenesis.

Toi et al. ${ }^{46}$ observed fine centripetal vasculature on the outer edge of the tumor with distorted vessels. Tumor-related blood vessels seem to converge from the normal breast tissue toward the center of the tumor, becoming drastically narrower at the tumor edge and nearly vanishing near the center [Fig. 10(d)]. Oraevsky et al. ${ }^{57}$ identified similar characteristic, which was described as areas of increased vascularity in the boundary and peripheral regions of the tumor.

Diot et al. $^{30}$ detected high peripheral vascularization in tumors along with a fivefold variability in the TBV gradient for different tumors, which may lead to classification of different tumor subtypes.

Finally, as has been demonstrated in US elastography, tumor lesions are harder than benign or healthy lesions. ${ }^{65}$ Lin et al. ${ }^{31}$ implemented elastography in PA imaging. As shown in Fig. 10(f), the tumor regions, which are more rigid, show relatively lower area change (blue) in comparison to surrounding normal breast tissue (light blue and yellow).

In addition to the general PA features, recent studies from the Pioneer $^{66}$ and Maestro ${ }^{67}$ clinical trials have shown that PA imaging can also identify different subtypes of breast cancer. The 


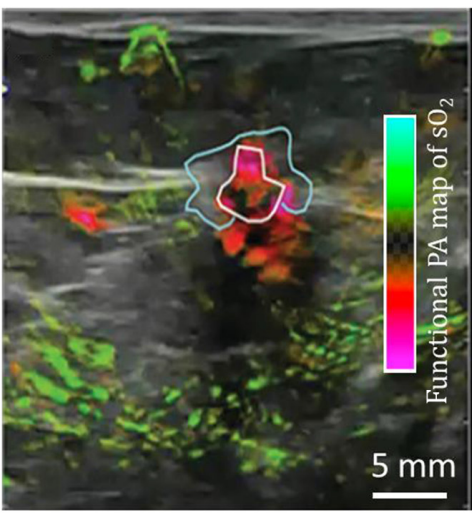

(a)

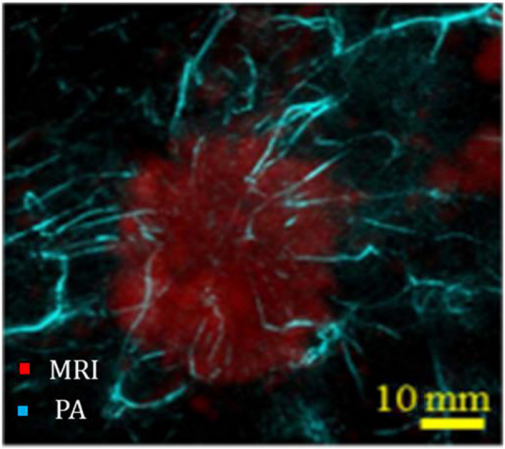

(d)

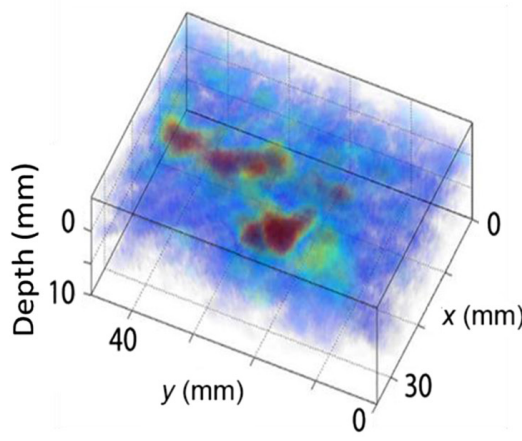

(b)

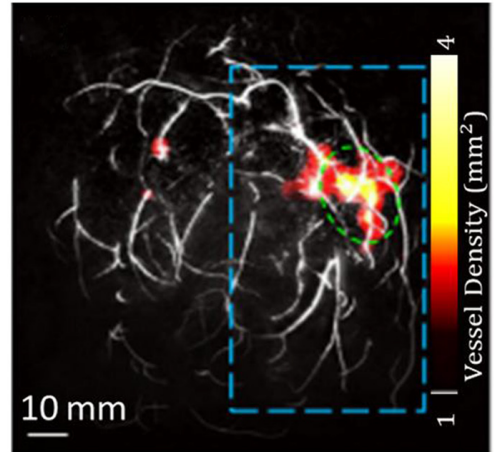

(e)

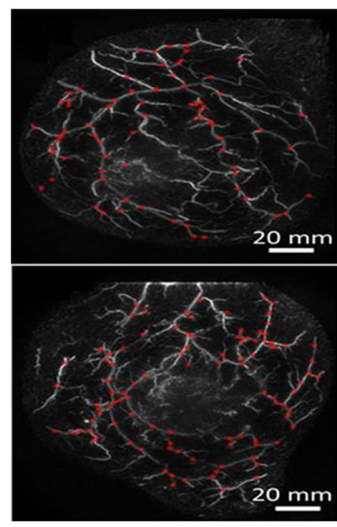

(c)

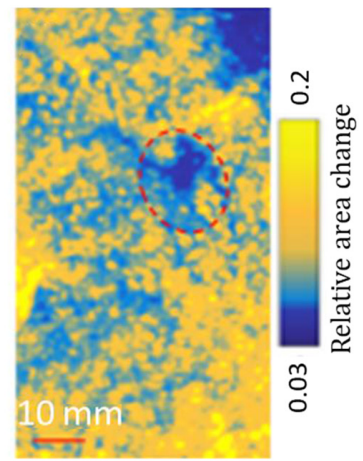

(f)

Fig. 10 PA features identified through various imaging systems. (a) Oxygen saturation: the PA combined map shows relatively oxygenated blood (green) and relatively deoxygenated blood (red) and is subjected to a threshold to minimize colorization of surrounding tissues. (C) 2017 RSNA. Reproduced with permission. ${ }^{66}$ (b) HbT concentration: image of $\mathrm{HbT}$ (high vascular density) in mucinous carcinoma of an 83-yearold patient. ${ }^{35}$ (c) VBPs represented as red dots that are identified using PA imaging show increased VBPs for breast with cancer (bottom) in comparison to healthy breast (top). ${ }^{45}$ (d) Centripetal vasculature: the PA features (cyan) are overlaid on MRI (red) image for a 47-mm tumor diameter in a 40-year-old woman with invasive breast carcinoma (IBC). The figures were acquired at a depth of $4 \mathrm{~mm}^{46}$ (e) Vessel density: vessel density map of breast with IDC of a 69-year-old female patient detected (green circle). Background gray scale image depicts the PA MAP image of vessels deeper than the nipple. (f) Relative area change: the PA elastographic image maps the relative area change during breathing in the regions outlined by blue dashed boxes in (e). Tumors are less compliant in comparison to normal breast tissue; therefore, they have smaller detectable relative area change when PA elastography is performed. The same tumor is identified by the red circle. ${ }^{31}$

four tumor subtypes ${ }^{68}$ are classified on the basis of estrogen or progesterone receptor, Ki-67 proliferation marker, and the HER2 (human epidermal growth factor receptor type 2) protein. The four tumor subtypes, luminal A (LUMA), luminal B (LUMB), triple negative breast cancer (TNBC), and human epidermal growth factor receptor type 2 enhanced (HER2-E), and their characteristics ${ }^{69-72}$ are presented in Table 3. Menezes et al $^{73}$ reviewed results from the Maestro trial, and Dogan et al. $^{74}$ reviewed results from the Pioneer trial to investigate whether tumor subtypes could be differentiated based on PA/US features. The studies found that the internal PA features (functional characteristics such as $\mathrm{Hb}, \mathrm{HbO}_{2}$, and $\mathrm{sO}_{2}$ ) and the peripheral vasculature played an important role in this classification process, along with the US feature of sound transmission (based on tumor anatomy ${ }^{75}$ ). Tumors with greater vascularity in the periphery and boundary zones and lesser internal features were typically subtype LUMA or LUMB. LUMB showed more internal features in comparison to LUMA; however, TNBCs were the richest in internal features with hypoxic characteristics. It was also found that HER2-E showed less peripheral features and more internal hypoxia; however, the internal features were sparse, similar to LUMA tumors. The features for HER2-E tumors could not be confirmed due to the small number of cases reported in the studies. These results are important as the subtype classification is an essential step in the determination of a treatment plan for the patient. With the help of PA imaging, the subtype classification may potentially be done noninvasively.

\section{Conclusion and Outlook}

This paper summarizes the current state-of-the-art PA imaging systems and the PA features identified for breast cancer detection. We noticed the following trends in PA imaging for breast cancer. First, obtaining functional information such as blood oxygen saturation and $\mathrm{HbT}$ concentration lend PA imaging the capability to noninvasively estimate tumor malignancy. As opposed to a single wavelength, multiple wavelengths can be used to map these characteristics to upgrade/downgrade masses for breast imaging reporting and data system classification. This gives a more accurate analysis of blood volume and hypoxia levels in the tumor site and also potentially avoids unnecessary 
Table 3 Tumor subtypes.

\begin{tabular}{|c|c|c|c|}
\hline Tumor subtype & Prognosis and treatment & US characteristics & PA characteristics \\
\hline $\begin{array}{l}\text { LUMA }=\mathrm{ER}^{+}, \mathrm{PR}^{+}, \mathrm{HER}^{-}(\mathrm{IHC} \\
0,1+\text {, or } 2+\text { with nonamplified } \\
\text { FISH), Ki- } 67<20 \%\end{array}$ & $\begin{array}{l}\text { Favorable, low histological grade, } \\
\text { and surgical or other oncological } \\
\text { treatments }\end{array}$ & $\begin{array}{l}\text { Irregular shape and prominent } \\
\text { posterior acoustic shadowing } \\
\text { (poor water content) }\end{array}$ & $\begin{array}{l}\text { Prominent external vessels, } \\
\text { peripheral radiating vessels, } \\
\text { and least internal features }\end{array}$ \\
\hline $\begin{array}{l}\mathrm{LUMB}=\mathrm{ER}^{+}, \mathrm{HER}^{-}, \\
\mathrm{Ki} 67 \geq 20 \% \text {; or }=\mathrm{ER}^{+}, \mathrm{HER} 2^{+} \\
(\mathrm{IHC} 3+\text { or } 2+\mathrm{FISH} \text { amplified), } \\
\text { irrespective of PR or Ki-67 status }\end{array}$ & $\begin{array}{l}\text { Favorable, but worse than LUMA, } \\
\text { high histological grade compared } \\
\text { to LUMA, lower histological grade } \\
\text { compared to TNBC, and surgical or } \\
\text { other oncological treatments }\end{array}$ & $\begin{array}{l}\text { Irregular shape and prominent } \\
\text { posterior acoustic shadowing } \\
\text { (poor water content) }\end{array}$ & $\begin{array}{l}\text { Prominent external vessels } \\
\text { and more internal vessels } \\
\text { than LUMA }\end{array}$ \\
\hline $\begin{array}{l}\text { Triple negative }(\mathrm{TNBC})=\mathrm{ER}^{-} \text {, } \\
\mathrm{PR}^{-}, \text {and HER2 }\end{array}$ & $\begin{array}{l}\text { Unfavorable, high histological grade, } \\
\text { and good response to neoadjuvant } \\
\text { chemotherapy }\end{array}$ & $\begin{array}{l}\text { Oval/round/well-circumscribed and } \\
\text { posterior acoustic enhancement } \\
\text { (water-rich, therefore, better } \\
\text { sound transmission) }\end{array}$ & $\begin{array}{l}\text { Lack of peripheral vessels } \\
\text { and internal vessel-rich in } \mathrm{Hb}\end{array}$ \\
\hline $\begin{array}{l}\text { HER2-enriched (HER2-E) }=\text { ER }^{-} \text {, } \\
\text { PR }^{-}, \text {HER2 }^{+}\end{array}$ & $\begin{array}{l}\text { Unfavorable, high histological grade, } \\
\text { and neoadjuvant chemotherapy }\end{array}$ & $\begin{array}{l}\text { Oval/round and posterior acoustic } \\
\text { enhancement }\end{array}$ & $\begin{array}{l}\text { Internal vessel-rich in } \mathrm{Hb} \\
\text { and less internal features in } \\
\text { comparison to TNBC }\end{array}$ \\
\hline
\end{tabular}

Note: ER, estrogen receptor; IHC, immunohistochemistry; PR, progesterone receptor; FISH, fluorescence in situ hybridization; HER2, human epidermal growth factor receptor type 2.

biopsies of benign tumors. Also other chromophores such as lipids, water, and melanin could be studied in the region of interest to further investigate tumor characteristics. ${ }^{30}$ However, adding more wavelengths may lead to an increase in system cost. Single-wavelength hemodynamic quantification methods, such as elastography and vessel density mapping, could be a more cost-effective solution. ${ }^{76,77}$ Second, with 3-D volumetric imaging instead of 2-D, vascular structures can be better identified. This also enables projection into different views that would allow better correlation with conventional imaging modalities in the clinic. Third, as a hybrid technique, most PA imaging systems can also perform US imaging. Combining PA and ultrasound images will offer both functional and anatomical information that can potentially enrich diagnosis. The PIONEER trial concluded that the PA/US imaging exceeded in specificity by $14.9 \%$ compared to internal US results of the system. ${ }^{66}$ The improvement is caused by the addition of functional characteristics from PA imaging results to the anatomical information obtained from US imaging. This meant that the addition of PA to US would potentially reduce the number of false-positives and reduce unnecessary biopsies of benign masses. Additional studies such as US tomography ${ }^{78,79}$ or elastography could be performed in conjunction with PA imaging to make the system more robust. Fourth, enhanced image quantification to improve visualization of PA features is necessary for aiding cancer detection. As shown in Table 2, some PA tumor features are not directly identifiable in the image. Computeraided detection, which has been widely adopted in radiology, ${ }^{80,81}$ could also be used for PA imaging to resolve features that are not obviously apparent. In addition, with machine learning and deep learning tools, background noise can be reduced in order to improve the quality of imaging results. ${ }^{82-84}$

PA imaging also has two major challenges. The first challenge is optical attenuation in tissue. Current results indicate that using multiple light illumination angles, along with optimizing imaging geometry can improve light penetration. ${ }^{34,51}$ In addition to that, engineering PA-based contrast agents could be another solution to facilitate deep tissue imaging. ${ }^{85-87}$ Second, although multispectral PA imaging enables the functional quantification of breast tissue, the complexity of light absorption and scattering in tissue make the quantification inaccurate. ${ }^{88}$ To this date, only the relative values of $\mathrm{HbO}_{2}, \mathrm{Hb}$, and $\mathrm{sO}_{2}$ have been reported. Therefore, these functional metrics need to be thoroughly investigated and standardized in order to pave the way for clinical translation. Along with existing results and continuous improvement in system designs and image reconstruction techniques, PA imaging holds great promise for clinical translation in the field of breast cancer screening and diagnosis.

\section{Disclosures}

The authors declare no competing interests.

\section{Acknowledgments}

This study was supported in part by the Career Catalyst Research Grant from the Susan G. Komen Foundation (No. CCR17481211). We would like to thank Melinda Tiao for her help with figures.

\section{References}

1. A. Jemal et al., "Global cancer statistics," CA Cancer J. Clin. 61(2), 69-90 (2011).

2. K. N. Nathanson, R. Wooster, and B. L. Weber, "Breast cancer genetics: what we know and what we need," Nat. Med. 7(5), 552-556 (2001).

3. R. Yerushalmi, M. Hayes, and K. A. Gelmon, "Breast carcinoma-rare types: review of the literature," Ann. Oncol. 20(11), 1763-1770 (2009).

4. S. R. Lakhani, "WHO classification of tumours of the breast," International Agency for Research on Cancer (2012).

5. H. P. Sinn and H. Kreipe, "A brief overview of the WHO classification of breast tumors, 4th Edition, focusing on issues and updates from the 3rd edition," Breast Care 8(2), 149-154 (2013).

6. U.-S. Albert et al., "Short version of the guideline: early detection of breast cancer in Germany," J. Cancer Res. Clin. Oncol. 130(9), 527536 (2004).

7. P. Boyle, "Global summit on mammographic screening," Ann. Oncol. 14(8), 1159-1160 (2003).

8. L. Tabár et al., "The natural history of breast carcinoma: what have we learned from screening?" Cancer 86(3), 449-462 (1999).

9. U.S. Preventive Services Task Force, "Screening for breast cancer: recommendations and rationale," Ann. Intern. Med. 137(5), 344 (2002).

10. R. W. Pinsky and M. A. Helvie, "Mammographic breast density: effect on imaging and breast cancer risk," J. Natl. Compr. Canc. Netw. 8(10), 1157-1165 quiz 1165 (2010). 
11. V. A. McCormack and I. D. S. Silva, "Breast density and parenchymal patterns as markers of breast cancer risk: a meta-analysis," Cancer Epidemiol. Biomarkers Prev. 15(6), 1159-1169 (2006).

12. C. K. Kuhl et al., "Mammography, breast ultrasound, and magnetic resonance imaging for surveillance of women at high familial risk for breast cancer," J. Clin. Oncol. 23(33), 8469-8476 (2005).

13. D. Ibrahim et al., "Heavy metal poisoning: clinical presentations and pathophysiology," Clin. Lab. Med. 26(1), 67-97 viii (2006).

14. K. J. Murphy, J. A. Brunberg, and R. H. Cohan, "Adverse reactions to gadolinium contrast media: a review of 36 cases," AJR Am. J. Roentgenol. 167(4), 847-849 (1996).

15. M. A. Perazella, "Gadolinium-contrast toxicity in patients with kidney disease: nephrotoxicity and nephrogenic systemic fibrosis," Curr. Drug Safety 3(1), 67-75 (2008).

16. W. A. Berg et al., "Detection of breast cancer with addition of annual screening ultrasound or a single screening MRI to mammography in women with elevated breast cancer risk," JAMA 307(13), 1394-1404 (2012).

17. R. F. Brem et al., "Assessing improvement in detection of breast cancer with three-dimensional automated breast US in women with dense breast tissue: the SomoInsight study," Radiology 274(3), 663-673 (2015).

18. N. Ohuchi et al., "Sensitivity and specificity of mammography and adjunctive ultrasonography to screen for breast cancer in the Japan Strategic Anti-cancer Randomized Trial (J-START): a randomised controlled trial," Lancet 387(10016), 341-348 (2016).

19. J. Xia, J. Yao, and L. V. Wang, "Photoacoustic tomography: principles and advances," Electromagn. Waves 147, 1-22 (2014).

20. L. V. Wang and H.-I. Wu, Biomedical Optics: Principles and Imaging, John Wiley \& Sons, Hoboken, New Jersey (2012).

21. P. Beard, "Biomedical photoacoustic imaging," Interface Focus 1(4), 602-631 (2011).

22. D. R. Bielenberg and B. R. Zetter, "The contribution of angiogenesis to the process of metastasis," Cancer J. 21(4), 267-273 (2015).

23. C. Hoelen et al., "Three-dimensional photoacoustic imaging of blood vessels in tissue," Opt. Lett. 23(8), 648-650 (1998).

24. J. Folkman, "Tumor angiogenesis: therapeutic implications," N. Engl. J. Med. 285(21), 1182-1186 (1971).

25. S. R. McDougall, A. R. Anderson, and M. A. Chaplain, "Mathematical modelling of dynamic adaptive tumour-induced angiogenesis: clinical implications and therapeutic targeting strategies," J. Theor. Biol. 241(3), 564-589 (2006).

26. B. J. Tromberg et al., "Assessing the future of diffuse optical imaging technologies for breast cancer management," Med. Phys. 35(6), 24432451 (2008).

27. M. H. Xu and L. H. V. Wang, "Universal back-projection algorithm for photoacoustic computed tomography," Phys. Rev. E 71(1), 016706 (2005).

28. R. A. Kruger et al., "Dedicated 3-D photoacoustic breast imaging," Med. Phys. 40(11), 113301 (2013).

29. X. L. Deán-Ben et al., "Volumetric hand-held optoacoustic angiography as a tool for real-time screening of dense breast," J. Biophotonics 9(3), 253-259 (2016).

30. G. Diot et al., "Multispectral optoacoustic tomography (MSOT) of human breast cancer," Clin. Cancer Res. 23(22), 6912-6922 (2017).

31. L. Lin et al., "Single-breath-hold photoacoustic computed tomography of the breast," Nat. Commun. 9(1), 2352 (2018).

32. X. Li et al., "High resolution functional photoacoustic tomography of breast cancer," Med. Phys. 42(9), 5321-5328 (2015).

33. D. Piras et al., "Photoacoustic imaging of the breast using the Twente Photoacoustic Mammoscope: present status and future perspectives," IEEE J. Sel. Top. Quantum Electron. 16(4), 730-739 (2010).

34. N. Nyayapathi et al., "Dual scan mammoscope (DSM) — a new portable photoacoustic breast imaging system with scanning in craniocaudal plane," IEEE Trans. Biomed. Eng. (2019).

35. M. Heijblom et al., "The state of the art in breast imaging using the Twente Photoacoustic Mammoscope: results from 31 measurements on malignancies," Eur. Radiol. 26(11), 3874-3887 (2016).

36. M. Heijblom et al., "Visualizing breast cancer using the Twente Photoacoustic Mammoscope: what do we learn from twelve new patient measurements?" Opt. Express 20(11), 11582-11597 (2012).

37. M. Heijblom et al., "Imaging breast lesions using the Twente Photoacoustic Mammoscope: ongoing clinical experience," Proc. SPIE 8223, 82230C (2012).
38. S. Manohar et al., "The Twente Photoacoustic Mammoscope: system overview and performance," Phys. Med. Biol. 50(11), 2543-2557 (2005).

39. S. Manohar et al., "Initial results of in vivo non-invasive cancer imaging in the human breast using near-infrared photoacoustics," Opt. Express 15(19), 12277-12285 (2007).

40. J. Xia et al., "Three-dimensional photoacoustic tomography based on the focal-line concept," J. Biomed. Opt. 16(9), 090505 (2011).

41. E. Fakhrejahani et al., "Clinical report on the first prototype of a photoacoustic tomography system with dual illumination for breast cancer imaging," PLoS One 10(10), e0139113 (2015).

42. Y. Asao et al., "Photoacoustic mammography capable of simultaneously acquiring photoacoustic and ultrasound images," J. Biomed. Opt. 21(11), 116009 (2016).

43. T. Kitai et al., "Photoacoustic mammography: initial clinical results," Breast Cancer 21(2), 146-153 (2014).

44. R. A. Kruger et al., "Photoacoustic angiography of the breast," Med. Phys. 37(11), 6096-6100 (2010).

45. I. Yamaga et al., "Vascular branching point counts using photoacoustic imaging in the superficial layer of the breast: a potential biomarker for breast cancer," Photoacoustics 11, 6-13 (2018).

46. M. Toi et al., "Visualization of tumor-related blood vessels in human breast by photoacoustic imaging system with a hemispherical detector array," Sci. Rep. 7, 41970 (2017).

47. Y. Matsumoto et al., "Visualising peripheral arterioles and venules through high-resolution and large-area photoacoustic imaging," Sci. Rep. 8(1), 14930 (2018).

48. J. Kruger et al., "Simulation of mammographic breast compression in 3-D MR images using ICP-based B-spline deformation for multimodality breast cancer diagnosis," Int. J. Comput. Assist. Radiol. Surg. 9(3), 367-377 (2014)

49. T. W. Sederberg and S. R. Parry, "Free-form deformation of solid geometric models," in Proc. 13th Annu. Conf. Comput. Graphics and Interactive Tech., ACM, pp. 151-160 (1986).

50. D. Baraff and A. Witkin, "Large steps in cloth simulation," in SIGGRAPH98 Conf. Proc., pp. 43-54 (1998).

51. A. Oraevsky et al., "Full-view 3-D imaging system for functional and anatomical screening of the breast," Proc. SPIE 10494, 104942Y (2018).

52. S. Park et al., "Compensation for non-uniform illumination and optical fluence attenuation in three-dimensional optoacoustic tomography of the breast," Proc. SPIE 10878, 108784X (2019).

53. S. L. Jacques, "Optical properties of biological tissues: a review (vol 58, pg R37, 2013)," Phys. Med. Biol. 58(14), 5007-5008 (2013).

54. D. F. Swinehart, "The Beer-Lambert Law," J. Chem. Educ. 39(7), 333 (1962).

55. L. Yao and H. Jiang, "Enhancing finite element-based photoacoustic tomography using total variation minimization," Appl. Opt. 50(25), 5031-5041 (2011).

56. L. Xi et al., "Design and evaluation of a hybrid photoacoustic tomography and diffuse optical tomography system for breast cancer detection," Med. Phys. 39(5), 2584-2594 (2012).

57. A. A. Oraevsky et al., "Clinical optoacoustic imaging combined with ultrasound for coregistered functional and anatomical mapping of breast tumors," Photoacoustics 12, 30-45 (2018).

58. A. A. Karabutov et al., "Backward mode detection of laser-induced wide-band ultrasonic transients with optoacoustic transducer," J. Appl. Phys. 87(4), 2003-2014 (2000).

59. X. L. Dean-Ben and D. Razansky, "Portable spherical array probe for volumetric real-time optoacoustic imaging at centimeter-scale depths," Opt. Express 21(23), 28062-28071 (2013).

60. X. L. Dean-Ben et al., "Accurate model-based reconstruction algorithm for three-dimensional optoacoustic tomography," IEEE Trans. Med. Imaging 31(10), 1922-1928 (2012).

61. A. Buehler et al., "Real-time handheld multispectral optoacoustic imaging," Opt. Lett. 38(9), 1404-1406 (2013).

62. A. Buehler et al., "Imaging of fatty tumors: appearance of subcutaneous lipomas in optoacoustic images," J. Biophotonics 10(8), 983-989 (2017).

63. A. Becker et al., "Multispectral optoacoustic tomography of the human breast: characterisation of healthy tissue and malignant lesions using a hybrid ultrasound-optoacoustic approach," Eur Radiol. 28(2), 602-609 (2018). 
64. D. J. Hicklin and L. M. Ellis, "Role of the vascular endothelial growth factor pathway in tumor growth and angiogenesis," J. Clin. Oncol. 23(5), 1011-1027 (2005).

65. B. Liu et al., "Breast lesions: quantitative diagnosis using ultrasound shear wave elastography-a systematic review and meta-analysis," Ultrasound Med. Biol. 42(4), 835-847 (2016).

66. E. I. Neuschler et al., "A pivotal study of optoacoustic imaging to diagnose benign and malignant breast masses: a new evaluation tool for radiologists," Radiology 287(2), 398-412 (2018).

67. G. L. G. Menezes et al., "Downgrading of breast masses suspicious for cancer by using optoacoustic breast imaging," Radiology 288(2), 355-365 (2018).

68. A. Goldhirsch et al., "Personalizing the treatment of women with early breast cancer: highlights of the St Gallen International Expert Consensus on the Primary Therapy of Early Breast Cancer 2013," Ann. Oncol. 24(9), 2206-2223 (2013).

69. P. Maisonneuve et al., "Proposed new clinicopathological surrogate definitions of luminal A and luminal B (HER2-negative) intrinsic breast cancer subtypes," Breast Cancer Res. 16(3), R65 (2014).

70. S. Badve et al., "Basal-like and triple-negative breast cancers: a critical review with an emphasis on the implications for pathologists and oncologists," Mod. Pathol. 24(2), 157-167 (2011).

71. R. B. Paschoalini et al., "Cytological criteria for predicting the luminal phenotype of breast carcinoma," Acta Cytol. 60(5), 406-412 (2016).

72. M. C. U. Cheang et al., "Ki67 Index, HER2 status, and prognosis of patients with luminal B breast cancer," JNCI J. Nat. Cancer Inst. 101(10), 736-750 (2009).

73. G. L. G. Menezes et al., "Optoacoustic imaging of the breast: correlation with histopathology and histopathologic biomarkers," Eur. Radiol. 1-13 (2019).

74. B. E. Dogan et al., "Optoacoustic imaging and gray-scale US features of breast cancers: correlation with molecular subtypes," Radiology 292(3), 564-572 (2019).

75. A. Irshad et al., "Assessing the role of ultrasound in predicting the biological behavior of breast cancer," AJR Am. J. Roentgenol. 200(2), 284-290 (2013)

76. A. Danielli et al., "Single-wavelength functional photoacoustic microscopy in biological tissue," Opt. Lett. 36(5), 769-771 (2011).

77. J. Yao et al., "High-speed label-free functional photoacoustic microscopy of mouse brain in action," Nat. Methods 12(5), 407-410 (2015).

78. J. Xia et al., "Enhancement of photoacoustic tomography by ultrasonic computed tomography based on optical excitation of elements of a fullring transducer array," Opt. Lett. 38(16), 3140-3143 (2013).
79. E. Mercep et al., "Transmission-reflection optoacoustic ultrasound (TROPUS) computed tomography of small animals," Light Sci. Appl. 8, 18 (2019).

80. J. J. Fenton et al., "Influence of computer-aided detection on performance of screening mammography," N. Engl. J. Med. 356(14), 13991409 (2007)

81. C. D. Lehman et al., "Diagnostic accuracy of digital screening mammography with and without computer-aided detection," JAMA Intern. Med. 175(11), 1828-1837 (2015).

82. S. Antholzer et al., "Deep learning for photoacoustic tomography from sparse data," Inverse Prob. Sci. Eng. 27(7), 987-1005 (2019).

83. D. Allman, A. Reiter, and M. A. L. Bell, "Photoacoustic source detection and reflection artifact removal enabled by deep learning," IEEE Trans. Med. Imaging 37(6), 1464-1477 (2018).

84. A. Hauptmann et al., "Model-based learning for accelerated, limitedview 3-D photoacoustic tomography," IEEE Trans. Med. Imaging 37(6), 1382-1393 (2018).

85. U. Chitgupi et al., "Surfactant-stripped micelles for NIR-II photoacoustic imaging through $12 \mathrm{~cm}$ of breast tissue and whole human breasts," Adv. Mater. 31, 1902279 (2019).

86. G. Ku et al., "Copper sulfide nanoparticles as a new class of photoacoustic contrast agent for deep tissue imaging at $1064 \mathrm{~nm}$," ACS Nano 6(8), 7489-7496 (2012).

87. Y. Gawale et al., "Carbazole-linked near-infrared Aza-BODIPY dyes as triplet sensitizers and photoacoustic contrast agents for deep-tissue imaging," Chemistry 23(27), 6570-6578 (2017).

88. M. Li, Y. Tang, and J. Yao, "Photoacoustic tomography of blood oxygenation: a mini review," Photoacoustics 10, 65-73 (2018).

Nikhila Nyayapathi graduated with BE (Hons) in Electronics and Instrumentation Engineering from Birla Institute of Technology and Science-Pilani, Dubai, in 2011. She earned her master's degree in Electrical Engineering from UB in 2016, and is currently pursuing her $\mathrm{PhD}$ in Electrical Engineering. She joined Dr. Xia's group in order to explore her interests in biomedical imaging and photoacoustics.

Jun Xia completed his $\mathrm{PhD}$ training in 2010 with Dr. Andreas Mandelis from the University of Toronto. In the same year, he joined Dr. Lihong V. Wang's lab at Washington University in St. Louis as a postdoctoral fellow. He joined University at Buffalo, the State University of New York in 2014 as an assistant professor. He has published three book chapters and more than sixty papers in the field of photoacoustic and photothermal imaging. 